\title{
Flexible power modeling of LTE base stations
}

\author{
Claude Desset ${ }^{1}$, Björn Debaillie ${ }^{1}$, Vito Giannini ${ }^{1}$, Albrecht Fehske ${ }^{2}$, Gunther Auer ${ }^{3}$, Hauke Holtkamp ${ }^{3}$, \\ Wieslawa Wajda ${ }^{4}$, Dario Sabella ${ }^{5}$, Fred Richter ${ }^{2}$, Manuel J. Gonzalez ${ }^{6}$, Henrik Klessig ${ }^{2}$, István Gódor ${ }^{7}$, \\ Magnus Olsson ${ }^{8}$, Muhammad Ali Imran ${ }^{9}$, Anton Ambrosy ${ }^{4}$, Oliver Blume ${ }^{4}$ \\ ${ }^{1}$ Imec, Kapeldreef, 75, B-3001 Leuven, Belgium \\ ${ }^{2}$ Technische Universität Dresden, 01062 Dresden, Germany \\ ${ }^{3}$ DOCOMO Euro-labs, Landsberger Str. 312, 80687 Munich, Germany \\ ${ }^{4}$ Alcatel-Lucent Deutschland AG, Lorenzstrasse 10, ZFZ/WA1, 70435 Stuttgart, Germany \\ ${ }^{5}$ Telecom Italia S.p.A. via G.Reiss Romoli 274, 10148 Turin, Italy \\ ${ }^{6}$ TTI Norte, S.L., Albert Einstein 14 PCTCAN, 39011 Santander, Cantabria \\ ${ }^{7}$ Ericsson Magyarorszg Kft. H-1037 Budapest, Laborc u. 1. Hungary \\ ${ }^{8}$ Ericsson AB, SE-164 80 Stockholm, Sweden \\ ${ }^{9}$ Centre for Comm. Systems Research (CCSR), BA Building, University of Surrey, Guildford, GU2 7XH, UK \\ contact author: desset@imec.be - +32.16.28.11.63
}

\begin{abstract}
With the explosion of wireless communications in number of users and data rates, the reduction of network power consumption becomes more and more critical. This is especially true for base stations which represent a dominant share of the total power in cellular networks. In order to study power reduction techniques, a convenient power model is required, providing estimates of the power consumption in different scenarios. This paper proposes such a model, accurate but simple to use. It evaluates the base station power consumption for different types of cells supporting the 3GPP LTE standard. It is flexible enough to enable comparisons between state-of-the-art and advanced configurations, and an easy adaptation to various scenarios. The model is based on a combination of base station components and sub-components as well as power scaling rules as functions of the main system parameters.
\end{abstract}

Index Terms-LTE, base station, power consumption, power model, energy efficiency, green radio

\section{INTRODUCTION}

In a world of exploding wireless communications, improving the power efficiency of radio networks is an important research topic [1]. To evaluate the energy efficiency of today's mobile communication systems and to identify improvement areas for next generation systems, a high level energy efficiency evaluation framework $\left(E^{3} F\right)$ has been developed within the Energy Aware Radio and neTwork tecHnologies (EARTH) project [2]. This framework [3] covers the complete system, including network and radios. It enables a quantitative evaluation in terms of efficiency under different traffic and load scenarios. The $\mathrm{E}^{3} \mathrm{~F}$ builds on state-of-the-art radio network evaluation methodology from system level simulations extended with power models, traffic models, and deployment models.

This paper ${ }^{1}$ describes the detailed power models of the base station components and sub-components and focuses on how the power is scaled over different scenarios. Four different types of base stations are considered: macro, micro, pico and femto cells. To increase the application area, power is used instead of energy because it is a more natural metric to use

\footnotetext{
${ }^{1}$ Acknowledgment: The research leading to these results has received funding from the European Community's Seventh Framework Programme FP7/2007-2013 under grant agreement n 247733 project EARTH.
}

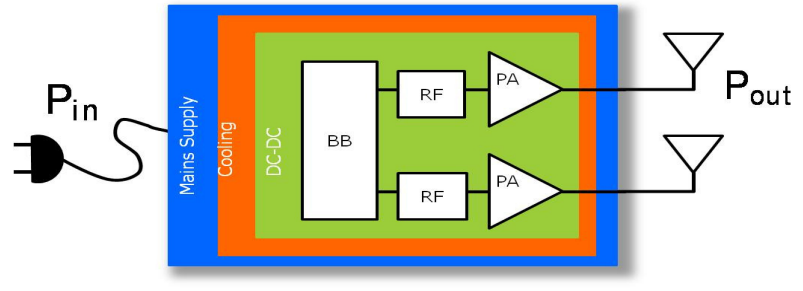

Fig. 1. Overview of base station components included in the power model. as a function of required throughput or system load, while energy is depending on a given amount of data to transmit in a batch but does not directly relate to system load and efficiency. The model has been designed for good accuracy, simplicity, and flexibility. It enables relative comparisons over many scenarios, even if fully accurate absolute power numbers cannot be guaranteed. This would require extensive physical measurements, and also prevent the model from being used to explore non-measured scenario.

Section II describes the general model structure and its parameters as visible to the model user. Section III details the model construction for all the main components of a typical base station. Section IV illustrates the way the model is used and shows the kind of results that can be obtained. Finally, Section V summarizes the model and its goals.

\section{MOdel STRUCTURE}

The power model is built around the split of a base station into a number of components and sub-components, as shown in Figure 1. This section introduces those components, distinguishes between different base station types, and presents the main parameters used to compute the power consumption in a specific scenario.

\section{A. Types of base stations and general parameters}

Four types of base stations are included in the model: macro, micro, pico, and femto. They are different in the subcomponents they contain as well as in the power figures associated with those sub-components, based on different constraints 
TABLE I

PARAMETERS AFFECTING SCALING OF BASEBAND AND RF POWER CONSUMPTION. DEFAULT VALUES CORRESPOND TO THE BASELINE CONFIGURATION OF THE BASE STATION. MODULATION IS EXPRESSED IN BITS PER SYMBOL, I.E., 1, 2, 4 OR 6 FOR BPSK, QPSK, 16-QAM OR 64-QAM, RESPECTIVELY.

\begin{tabular}{|c|c|c|c|}
\hline Notation & Description & Range & Default \\
\hline$B W$ & Bandwidth $[\mathrm{MHz}]$ & $1.4-20$ & 10 \\
\hline$A n t$ & Number of antennas & $1,2,4$ & 2 \\
\hline$M$ & Modulation & $1,2,4,6$ & 6 \\
\hline$R$ & Coding rate & $1 / 3-1$ & $5 / 6$ \\
\hline$d t$ & Time-domain duty-cycling & $0-1$ & 1 \\
\hline$d f$ & Frequency-domain duty-cycling & $0-1$ & 1 \\
\hline
\end{tabular}

(output power, maximum load, signal accuracy...). They translate into different basic architectures, for example small base stations (pico and femto) may use more power-efficient dedicated components while large base stations (macro and micro) require more reconfigurability, for example using more FPGAs and less dedicated hardware. This translates into different intrinsic power efficiencies.

Macro base stations are also characterized in the model by having a variable number of sectors while other base station types only have one. The four base station types also differ in maximum output power.

Next to the base station type, the silicon technology is a general parameter affecting the power consumption obtained from the model. It can be specified as either CMOS feature size in $\mathrm{nm}$ or year of deployment. The default value for technology is $65 \mathrm{~nm}$ or 2010 deployment.

The remaining general parameters define the losses of different sub-components related to the power systems of the base station. This includes losses from cooling, AC/DC conversion, DC/DC conversion and antenna feeder. The three first terms are described in Subsection III-D. Antenna feeder losses are only present between the PA and the antenna in macro base stations. They are included together with the PA model. Other base station types do not have feeder losses due to their more compact design.

\section{B. Base station sub-components and scaling parameters}

The sub-component split is based on both real hardware and architecture splits, e.g., analog RF part versus digital baseband part, as well as functional splits, e.g., time-domain processing versus frequency-domain processing. Architecture splits include digital baseband, RF (analog), power amplifier, and overhead (power systems and cooling):

$$
P_{\text {Total }}=P_{\mathrm{BB}}+P_{\mathrm{RF}}+P_{\mathrm{PA}}+P_{\text {Overhead }}
$$

The reason for those splits is twofold: making clear links to the power consumption of specific components in the system in order to get reference numbers but also identifying how each power figure scales with parameters (time-domain computations may not scale the same way as frequencydomain computations when changing the modulation or system load). As a consequence, the model is centered around two types of tables. Power tables contain reference power figures that were obtained from measurements for the different subcomponents of a base station and reasoning on the architecture and specifications of the different types of base stations (see Tables II, III, V and VI). Scaling tables contain specific scaling factors telling how each power figure evolves with each specific parameter (see Table IV and Section III-B).

This approach is used for baseband as well as RF power consumption. Defining $I_{\mathrm{BB}}$ the set of baseband subcomponents, $I_{\mathrm{RF}}$ the set of analog sub-components, and $X=$ $\{B W, A n t, M, R, d t, d f\}$ the list of parameters as described in Table I, we get the following expression ${ }^{2}$ :

$$
\begin{aligned}
P_{\text {Total }} & =\sum_{i \in I_{\mathrm{BB}}} P_{i, \text { ref }} \prod_{x \in X}\left(\frac{x_{\mathrm{act}}}{x_{\mathrm{ref}}}\right)^{s_{i, x}} \\
& +\sum_{i \in I_{\mathrm{RF}}} P_{i, \text { ref }} \prod_{x \in X}\left(\frac{x_{\mathrm{act}}}{x_{\text {ref }}}\right)^{s_{i, x}} \\
& +P_{\mathrm{PA}}+P_{\text {Overhead }}
\end{aligned}
$$

The number of antennas is assumed to be the same in transmission and in reception, and also the same as the number of spatial streams. Time-domain duty-cycling represents the fraction of time during which the base station is operating, assuming it fully sleeps during the rest of the time. It is indeed an interesting technique in order to reduce the average power consumption [4]. However, depending on the hardware design and on the speed at which transitions between active and sleeping states should be performed, the full sleeping assumption may be too optimistic, as further discussed in Section IV. Frequency-domain duty-cycling represents the fractional load of the system in frequency resources, i.e., PRBs or physical resource blocks.

Scaling factors are exponents relating each power contributor in the model to each parameter of the model. For example, if the power of a mixer is not impacted by the number of resource blocks currently in use, the exponent will be zero, meaning that a change in resource block allocation (frequencydomain duty-cycling) has no influence on the power of that mixer. Similarly, the baseband demapping complexity will scale linearly with the number of loaded resource blocks, so the corresponding exponent will be one. Other values than zero and one are occasionally used, for example in order to model the quadratic or cubic MIMO processing complexity as function of the number of antennas.

For digital computations, we use complexity figures (GOPS or Giga Operations Per Second) that can be translated into power figures depending on the intrinsic efficiency of the selected technology (GOPS/W). Although this is a crude approximation, this is the only one compatible with the simplicity and flexibility of the model. The power numbers obtained from this estimation have been benchmarked to actual values in existing base stations in order to validate the approach and ensure meaningful results.

\section{MODEL COMPONENTS}

This section details the power models of the different components of a base station, i.e., the digital baseband, the analog RF, the power amplifier and the power system (power conversion and cooling). The corresponding power figures have been derived from existing designs.

\footnotetext{
${ }^{2}$ Baseband sub-components are listed in Table II; RF sub-components are listed in Tables V and VI.
} 
TABLE II

COMPLEXITY OF BASEBAND OPERATIONS IN DOWNLINK (GOPS IN REFERENCE SCENARIO).

\begin{tabular}{|c|c|c|c|c|}
\hline GOPS per operation type & Macro & Micro & Pico & Femto \\
\hline DPD & 160 & 160 & 0 & 0 \\
\hline Filter & 200 & 160 & 120 & 100 \\
\hline CRPI/SERDES & 360 & 300 & 0 & 0 \\
\hline OFDM & 80 & 80 & 70 & 60 \\
\hline FD (linear) & 30 & 30 & 20 & 20 \\
\hline FD (non-linear) & 10 & 10 & 5 & 5 \\
\hline FEC & 20 & 20 & 20 & 20 \\
\hline CPU & 200 & 200 & 30 & 20 \\
\hline
\end{tabular}

\section{A. Digital baseband processing}

The digital processing is modeled based on estimated complexity in GOPS, multiplied by a technology-dependent factor expressing the number of operations that can be perfomed per second and per Watt. This factor is $40 \mathrm{GOPS} / \mathrm{W}$ for large base stations and default technology, i.e., $65 \mathrm{~nm}$ General Purpose CMOS. It is three times larger for pico and femto cells given the more dedicated hardware used, as discussed in Subsection II-A. The digital complexity is split into a number of sub-components:

- DPD: Digital Pre-Distortion

- Filter: up/down-sampling and filtering

- CRPI/SERDES: serial link to backbone network

- OFDM: FFT and OFDM-specific processing

- FD: Frequency-Domain processing (mapping/demapping, MIMO equalization); it is split into two parts, scaling linearly and non-linearly with the number of antennas

- FEC: Forward Error Correction

- CPU: platform control processor

The non-linear part of frequency-domain processing accounts for the MIMO operations with quadratic or cubic scaling. The relatively small contributions for frequency-domain processing in Table II (single-antenna reference case) can hence make up a more significant share of the total power with 2 or 4 antennas.

Next to those terms, the leakage power is also taken into account, leading the following total baseband power consumption:

$$
\begin{aligned}
P_{\mathrm{BB}} & =P_{\mathrm{Dynamic}}+P_{\mathrm{Leak}}, \text { where } \\
P_{\mathrm{Dynamic}} & =P_{\mathrm{DPD}}+P_{\mathrm{Filter}}+P_{\mathrm{OFDM}}+P_{\mathrm{FD}, \text { lin }} \\
& +P_{\mathrm{FD}, \mathrm{nl}}+P_{\mathrm{CRPI}}+P_{\mathrm{FEC}}+P_{\mathrm{CPU}}
\end{aligned}
$$

The reference leakage power is defined as function of the reference dynamic power, where $\eta_{\text {Leak }}$ is 0.1 in $65 \mathrm{~nm}$ technology.

$$
P_{\text {Leak,Ref }}=\eta_{\text {Leak }} P_{\text {Dynamic,Ref }}
$$

Both dynamic and leakage power have their own scaling rules with technology. We assume for each CMOS generagion, e.g., moving from $65 \mathrm{~nm}$ to $45 \mathrm{~nm}$, a two-fold reduction of the dynamic power but a three-fold worsening of leakage power, i.e., $\eta_{\text {Leak }}$ is multiplied by 3 [5]. This can be used to assess the power for $45 \mathrm{~nm}$ (2012 deployment) instead of the default $65 \mathrm{~nm}$ in 2010 .

The complexity values given in Table II are used as reference case for the baseband in downlink. This reference case assumes $20 \mathrm{MHz}$ bandwidth, single-antenna, 64-QAM, rate-
TABLE III

COMPLEXITY OF BASEBAND OPERATIONS IN UPLINK (GOPS).

\begin{tabular}{|c|c|c|c|c|}
\hline GOPS per operation type & Macro & Micro & Pico & Femto \\
\hline Filter & 200 & 160 & 160 & 150 \\
\hline CRPI/SERDES & 360 & 300 & 0 & 0 \\
\hline OFDM & 80 & 80 & 80 & 60 \\
\hline FD (linear) & 60 & 60 & 40 & 30 \\
\hline FD (non-linear) & 20 & 20 & 10 & 10 \\
\hline FEC & 120 & 120 & 120 & 110 \\
\hline CPU & 200 & 200 & 30 & 20 \\
\hline
\end{tabular}

TABLE IV

SCALING EXPONENTS FOR THE BASEBAND SUB-COMPONENTS IN DOWNLINK (PARAMETERS ARE DEFINED IN SECTION II-B).

\begin{tabular}{|c|c|c|c|c|c|c|}
\hline Digital scaling exponents & $\mathrm{BW}$ & $\mathrm{M}$ & $\mathrm{R}$ & $\mathrm{Ant}$ & $\mathrm{dt}$ & $\mathrm{df}$ \\
\hline DPD, Filter and OFDM & 1 & 0 & 0 & 1 & 1 & 0 \\
\hline CRPI/SERDES & 1 & 1 & 1 & 1 & 1 & 1 \\
\hline FD (linear) & 1 & 0 & 0 & 1 & 1 & 1 \\
\hline FD (non-linear) & 1 & 0 & 0 & 2 & 1 & 1 \\
\hline FEC & 1 & 1 & 1 & 1 & 1 & 1 \\
\hline CPU & 0 & 0 & 0 & 1 & 0 & 0 \\
\hline Leakage & 1 & 0 & 0 & 1 & 0 & 0 \\
\hline
\end{tabular}

1 encoding $^{3}$ and a load ${ }^{4}$ of $100 \%$. This reference scenario is used within the model and its tables; it should not be confused with the baseline scenario representing the default operation of a base station as presented in Table I and illustrated in Section IV. The reference CMOS technology is $65 \mathrm{~nm}(2010$ reference year). Values are given in GOPS for the different types of base stations. The figures come from a mixture of system knowledge - estimating the relative complexity of different sub-components - and base station design expertise - matching the total power for selected technology options to the known order of magnitude in existing designs.

Some of the differences between larger and smaller base stations can be explained as follows:

- Predistortion is not applied to small base stations given the different type of PA and power levels.

- Filtering and signal accuracy constraints are more stringent for large base stations, with impact on both up/downsampling filters and MIMO-OFDM processing.

- The network link is done differently for small base stations (no specific backbone network, general IP connection instead).

- The platform control overhead is larger for a large base station due to the larger platform size including specific additional components.

The corresponding uplink numbers are provided in Table III. Most functional blocks are similar to the downlink case (Table II) but doing reverse operations (the filter is performing down-conversion in uplink, FEC is decoding...). Comparing to the downlink cases, we can note the following differences:

- Predistortion is not used in uplink.

- MIMO processing (equalization and detection) is more complex than the corresponding transmitter steps.

- FEC decoding is significantly more complex (for turbo or convolutional) than encoding.

${ }^{3}$ In reality the channel coding is deactivated when the rate is equal to 1 , but the corresponding value is used as reference for the largest possible throughput.

${ }^{4}$ The load is defined as fractional use of time and frequency resources, i.e., load $=d t \times d f$. 
TABLE V

RF ANALOG COMPONENT POWER $(m W)$, TRANSMITTER CASE (DOWNLINK).

\begin{tabular}{|c|c|c|c|c|}
\hline Power per analog component $[\mathrm{mW}]$ & Macro & Micro & Pico & Femto \\
\hline IQ modulator & 1000 & 1000 & 1000 & 1000 \\
\hline Variable attenuator & 10 & 10 & 0 & 0 \\
\hline Buffer & 300 & 300 & 0 & 0 \\
\hline Forward Voltage-Contr. Osc. (VCO1) & 170 & 170 & 170 & 170 \\
\hline Feedback Voltage-Contr. Osc. (VCO2) & 170 & 170 & 0 & 0 \\
\hline Feedback mixer & 1000 & 1000 & 0 & 0 \\
\hline Clock generation and buffering & 990 & 990 & 990 & 990 \\
\hline Digital-to-Analog Converter (DAC) & 1370 & 1370 & 200 & 200 \\
\hline Analog-to-Digital Converter (ADC) & 730 & 730 & 140 & 140 \\
\hline \hline Predriver (not incl. in total) & 2250 & 2250 & 0 & 0 \\
\hline \hline TOTAL (w/o predriver) & 5740 & 5740 & 2500 & 2500 \\
\hline Downscaling factor & 1 & 2 & 7 & 12 \\
\hline Total after downscaling [W] & 5.7 & 2.9 & .4 & .2 \\
\hline
\end{tabular}

Table IV gives the scaling exponents in downlink for each baseband sub-component and each scaling parameter. This table havs been derived by analyzing whether the amount of computations to perform in each sub-component was depending on each of the scaling parameters or not. DPD, filtering and OFDM are put together as time domain processing category, with the same scaling properties. Leakage power is also a category of its own. Clock gating is assumed when dutycycling components in time domain, but not power gating (leakage remains). In uplink, all exponents are the same, except for the non-linear frequency-domain processing (cubic dependency - exponent 3 - in uplink instead of the quadratic dependency - exponent 2 - in downlink).

Example of baseband power scaling: As illustration, let us assume we want to compute the power of frequency-domain, linear processing in case of 10-MHz, 2x2 MIMO, 16-QAM, coding rate $3 / 4,100 \%$ of time-domain duty-cycling and $30 \%$ of frequency occupation, we start from the reference scenario (20-MHz, single-antenna, 64-QAM, coding rate 1, 100\% timedomain and frequency-domain duty-cycling), leading for a macrocell in uplink to 60 GOPS (Table III) or $P_{\mathrm{FD}, \text { lin,ref }}=$ $1.5 \mathrm{~W}$ (with $40 \mathrm{GOPS} / \mathrm{W}$ in $65 \mathrm{~nm}$ ). The scaling vector with respect to the 6 parameters is $\left[s_{1}, s_{2} \cdots s_{6}\right]=[1,0,0,1,1,1]$ (Table IV $)^{5}$. It is applied to the ratio of all parameters between actual and reference scenarios, leading the actual power consumption of linearly-scaling frequency-domain processing:

$$
\begin{aligned}
P_{\mathrm{FD}, \text { lin }}= & P_{\mathrm{FD}, \text { lin, ref }}\left(\frac{B W_{\mathrm{act}}}{B W_{\mathrm{ref}}}\right)^{s_{1}}\left(\frac{M_{\mathrm{act}}}{M_{\mathrm{ref}}}\right)^{s_{2}}\left(\frac{R_{\mathrm{act}}}{R_{\mathrm{ref}}}\right)^{s_{3}} \\
& \left(\frac{A n t_{\mathrm{act}}}{A n t_{\mathrm{ref}}}\right)^{s_{4}}\left(\frac{d t_{\mathrm{act}}}{d t_{\mathrm{ref}}}\right)^{s_{5}}\left(\frac{d f_{\mathrm{act}}}{d f_{\mathrm{ref}}}\right)^{s_{6}} \\
= & 1.5 \mathrm{~W} \times\left(\frac{10}{20}\right)^{1}\left(\frac{4}{6}\right)^{0}\left(\frac{3 / 4}{1}\right)^{0}\left(\frac{2}{1}\right)^{1} \\
& \left(\frac{100}{100}\right)^{1}\left(\frac{30}{100}\right)^{1} \\
= & 0.45 \mathrm{~W}
\end{aligned}
$$

\section{B. RF sub-components}

The RF architecture contains the different elements of a low-IF/zero-IF architecture, in particular clock/carrier gener-

\footnotetext{
${ }^{5}$ Formally the scaling exponents are named $\left[s_{\mathrm{FD}, \text { lin,BW }} \cdots s_{\mathrm{FD}, \mathrm{lin}, \mathrm{df}}\right]$ according to (2). The shorter notation $\left[s_{1} \cdots s_{6}\right]$ has been selected in order to have a more readable equation (6).
}

TABLE VI

RF ANALOG COMPONENT POWER $(m W)$, RECEIVER CASE (UPLINK).

\begin{tabular}{|c|c|c|c|c|}
\hline Power per analog component $[\mathrm{mW}]$ & Macro & Micro & Pico & Femto \\
\hline First Low-Noise Ampl. (LNA1) & 300 & 300 & 300 & 300 \\
\hline Main variable attenuator & 10 & 10 & 10 & 10 \\
\hline Second Low-Noise Ampl. (LNA2) & 1000 & 1000 & 0 & 0 \\
\hline Dual mixer & 1000 & 1000 & 1000 & 1000 \\
\hline Dual IF Variable Gain Ampl. (VGA) & 650 & 650 & 0 & 0 \\
\hline Clock generation and buffering & 990 & 990 & 990 & 990 \\
\hline Analog-to-Digital Conv. (ADC) & 1190 & 1190 & 290 & 290 \\
\hline \hline TOTAL & 5140 & 5140 & 2590 & 2590 \\
\hline Downscaling factor & 1 & 2 & 7 & 12 \\
\hline Total after downscaling [W] & 5.1 & 2.6 & .4 & .2 \\
\hline
\end{tabular}

ation and distribution, modulator, mixers, low-noise amplifier (LNA), variable-gain amplifier (VGA), analog/digital converters (DAC and ADC), filters, buffer and predriver, and feedback chain. Only the PA is considered separately (Section III-C) because it cannot be captured in the same modeling approach. All the RF elements consume a specific amount of power, as given in Table $\mathrm{V}$ for downlink in reference technology of $65 \mathrm{~nm}$ CMOS.

The predriver is shown for information but included into the PA power. Some of the sub-components are not present in smaller base stations. Moreover, an overall downscaling factor reduces the power on smaller base stations due to less constraining specs and different hardware implementation of of smaller cells. The reason is two-fold. Firstly, the amount of blockers a small base station has to face is smaller, leading to more relaxed linearity specs and hence less power is needed. Secondly, smaller base stations can work from a lower supply voltage, further reducing their consumption.

Scaling of RF power with input parameters is done as follows. All RF sub-components have a scaling exponent 1 with respect to number of antennas and time-domain dutycycling. For carrier and clock generation sub-components, all the other scaling exponents have the value zero. For the other RF sub-components, the scaling exponent is also 1 with respect to frequency-domain duty-cycling, assuming a scalable implementation, and for small base stations with respect to bandwidth as well.

Technology scaling is used for analog, too, based on a scaling factor as function of the selected CMOS technology compared to the reference tech $=65 \mathrm{~nm}$ case. This is an empirical rule from designers' experience, not a physical law:

$$
\operatorname{Power}(\text { tech })=\operatorname{Power}(65 \mathrm{~nm})\left(1+\frac{\text { tech } / 65-1}{2}\right)
$$

Table VI provides the numbers for uplink direction, when the analog front-end is receiving. The same downscaling factors are used as in the downlink case.

\section{Power amplifier}

The power amplifier behavior cannot be captured by a single reference power number and scaling rules. Hence, the PA model is represented by a table containing measurements of output power versus consumed power. Measured points differ in requested output power and in tuning of the $1 \mathrm{~dB}$ compression point. The power model picks up the point with minimal power consumption that is satisfying the output power and linearity constraints. 

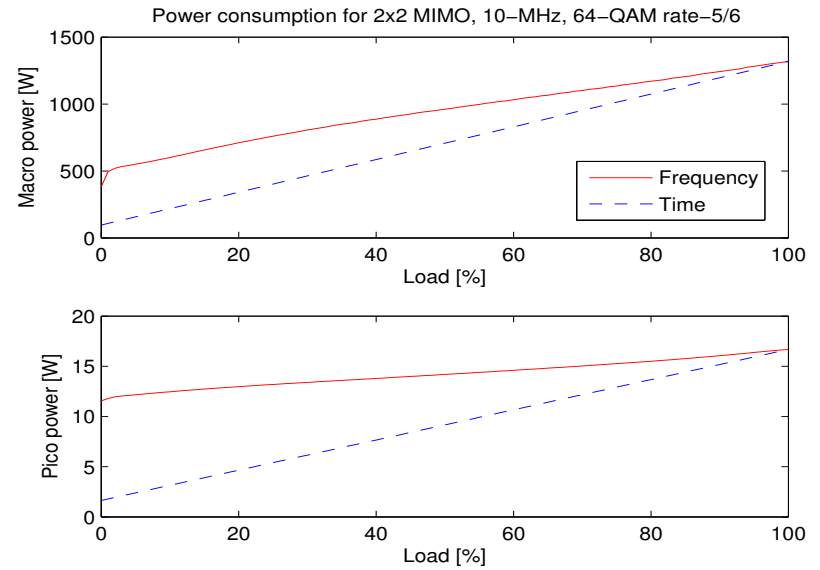

Fig. 2. Power consumption as function of load for a 3-sector macro cell and a pico cell. Load dependency is based on either frequency-domain duty-cycling or time-domain duty-cycling. FDD is expected (uplink plus downlink).

The maximum total output power is $46 \mathrm{dBm}$ for macro, $41 \mathrm{dBm}$ for micro, $24 \mathrm{dBm}$ for pico and $20 \mathrm{dBm}$ for femto cells. Additionally, feeder cable losses amount to $3 \mathrm{~dB}$ in macro base stations only. When using multiple antennas, this output power is divided between the antennas, keeping the same total value (within one sector). Large base station only have to support a PAPR of $8 \mathrm{~dB}$ thanks to the pre-distortion; small base stations have to support a PAPR of $12 \mathrm{~dB}$.

The PA selected for macro and micro base stations can output up to $54 \mathrm{dBm}$ at $1 \mathrm{~dB}$ compression for a consumption of $390 \mathrm{~W}$. Output power reduces linearly with frequency-domain load or explicit power control. The reduction in consumed power is not proportional given that the PA efficiency degrades at reduced load. The PA minimal power consumption at low output power is $11 \mathrm{~W}$. Similarly, small cells (pico and femto) use a PA design capable of up to $36 \mathrm{dBm}$ for a consumption of $8 \mathrm{~W}$. Its minimum power consumption is $650 \mathrm{~mW}$.

\section{Overhead}

In the overhead or power system category, we place all the sub-components that are related to the system powering, including AC/DC and DC/DC conversion as well as cooling. In order to keep a simple model, the power is computed as a fixed overhead linearly depending on the total power of the rest of the base station. The corresponding factors can be given as input parameters (otherwise default values are used, as specified in Section II-A):

$$
\begin{aligned}
P_{\text {overhead }} & =\left(P_{\mathrm{BB}}+P_{\mathrm{RF}}+P_{\mathrm{PA}}\right) \\
\times & \left(\left(1+\eta_{\mathrm{cool}}\right)\left(1+\eta_{\mathrm{dcdc}}\right)\left(1+\eta_{\mathrm{acdc}}\right)-1\right)
\end{aligned}
$$

Default values for the linear loss model are $10 \%$ for cooling but in macro base stations only, 5\% for DC/DC conversion, and $10 \%$ for AC/DC conversion. There is no cooling for smaller base stations.

\section{Model USAGE AND RESUlts}

The model has been implemented in Matlab. This enables a flexible generation of output power for different scenarios. All input parameters described in Section II are implemented including their default values for the baseline scenario. The

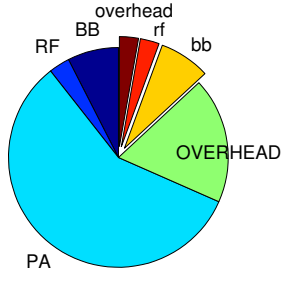

Macro (1320 W)

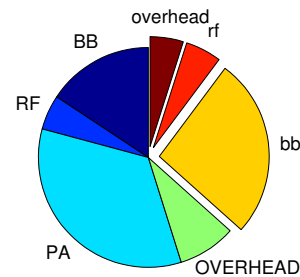

Pico $(17$ W)
Fig. 3. Relative share of the different components for two types of base stations (configuration of Figure 2). UPPERCASE labels denote DOWNLINK; lowercase labels and shares shifted out of the pie center denote uplink.

output separates power consumption in uplink and downlink, for each of the main components described in Section III.

Figure 2 illustrates the power obtained model as function of the system load. Two of the four base station types have been selected as example. Time-domain duty-cycling outperforms frequency-domain duty-cycling due to the possibility to let specific components sleep during inactive periods. However, the way time-domain duty-cycling it is implemented in the model and illustrated in this Figure is optimistic in the sense that duty-cycled components are assumed to be switched on and off without any overhead. This suits scenarios where sufficiently long sleeping time is present between transitions. When considering micro-sleeping for which this assumption does not hold, more realistic numbers are obtained by using the sleep mode approach of [3].

In the case of a macro-cell, $87 \%$ of the power is used for downlink and $13 \%$ for uplink. The relative share of the different components is shown on Figure 3. This illustrates that while large base stations are strongly dominated by PA and to a lesser extent by the power systems (overhead), smaller base stations are less PA-dominated and also contain a significant baseband share.

\section{Conclusions}

In this paper, we propose a high-level power consumption model for LTE base stations. It covers various types of base stations and is flexible due to a large number of parameters. It provides power figures for the different sub-components present in a typical base station. This model is mostly designed not to give very accurate absolute figures but to enable exploring different architectures as proposed within exploration projects (more antennas, trade-offs between macro and small cells, load impact, role of duty-cycling...).

\section{REFERENCES}

[1] A. Fehske, G. Fettweis, J. Malmodin, and G. Biczok, "The global footprint of mobile communications: The ecological and economic perspective," IEEE Communications Magazine, vol. 49, no. 8, pp. 55-62, Aug. 2011.

[2] "EARTH project under european community's seventh framework programme FP7/2007-2013, grant agreement n 247733," .

[3] Gunther Auer, Vito Giannini, István Gódor, Per Skillermark, Magnus Olsson, Muhammad Ali Imran, Dario Sabella, Manuel J. Gonzalez, Claude Desset, Oliver Blume, and Albrecht Fehske, "How much energy is needed to run a wireless network?," IEEE Wireless Communications Magazine, special issue on Technologies for Green Radio Communication Networks, vol. 18, no. 4, Oct. 2011.

[4] P. Frenger et al., "Reducing energy consumption in LTE with cell DTX," in IEEE VTC Spring, Budapest, Hungary, May 2011.

[5] Y. Taur, "CMOS design near the limit of scaling," IBM Journal of Research and Development, vol. 46, no. 2.3, pp. 213 -222, march 2002. 